\title{
PROPRIEDADES REDOX DA MATÉRIA ORGÂNICA ISOLADA DE MATERIAL ULTRAFILTRADO DAS ÁGUAS DO RIO PARAÍBA DO SUL
}

\author{
Marihus Altoé Baldotto*, Luciano Pasqualoto Canellas e Ary Carlos Xavier Velloso \\ Laboratório de Solos, Centro de Ciências e Tecnologias Agropecuárias, Universidade Estadual do Norte Fluminense Darcy Ribeiro, \\ Av. Alberto Lamego, 2000, 28013-602 Campos dos Goytacazes - RJ, Brasil \\ Raul Castro Carriello Rosa, Marcelo dos Santos Maciel e Carlos Eduardo Rezende \\ Laboratório de Ciências Ambientais, Centro de Biociências e Biotecnologia, Universidade Estadual do Norte Fluminense Darcy \\ Ribeiro, Av. Alberto Lamego, 2000, 28013-602 Campos dos Goytacazes - RJ, Brasil

\section{Maria Cristina Canela} \\ Laboratório de Ciências Químicas, Centro de Ciências e Tecnologias, Universidade Estadual do Norte Fluminense Darcy Ribeiro, \\ Av. Alberto Lamego, 2000, 28013-602 Campos dos Goytacazes - RJ, Brasil
}

Recebido em 4/4/08; aceito em 10/11/08; publicado na web em 6/3/09

\begin{abstract}
REDOX PROPERTIES OF ORGANIC MATERIAL PRESENT IN ULTRAFILTRATED WATER SAMPLES TAKEN FROM THE PARAÍBA DO SUL RIVER, BRAZIL. Previous studies indicated that free radicals control organic matter redox activities. In the present study, organic matter of an ultra-filtrated material collected from seven samples taken seasonally from the Paraiba do Sul River for two years were titrated with an oxidizer $\left(I_{2}\right)$ in an inert atmosphere. Standard formal potential values for the electrode $\mathrm{MO}_{\mathrm{Ox}}, \mathrm{MO}_{\mathrm{Red}}$ ranged from 0.754 to $0.786 \mathrm{~V}$ at a $25^{\circ} \mathrm{C}$ temperature. Organic matter oxidation capacity (COx) per carbon mass varied according to $\mathrm{pH}$ values, and changes in COx were related to rainfall and river flow intensities.
\end{abstract}

Keywords: redox potential; oxidation capacity; ultra-filtration.

\section{INTRODUÇÃO}

As titulações redox de ácidos húmicos $(\mathrm{AH})$ permitem estimar o potencial formal padrão do eletrodo $\left({ }^{\mathrm{F}} \mathrm{E}_{\mathrm{H}}{ }^{\circ}\right)$ e sua capacidade de oxidação ( $\mathrm{COx})$, fatores de intensidade e capacidade da atividade de elétrons, respectivamente. Estes parâmetros são análogos ao $\mathrm{pH}$ e à capacidade de neutralização para o caso de prótons. ${ }^{1,2} \mathrm{O}^{\mathrm{F}} \mathrm{E}_{\mathrm{H}}{ }^{\mathrm{o}}$ pode ser medido utilizando-se um eletrodo combinado, constituído de um eletrodo redox em contato com um eletrodo de referência. O valor do ${ }^{\mathrm{F}} \mathrm{E}_{\mathrm{H}}{ }^{\circ}$ possibilita a determinação das atividades de oxidantes e redutores para um determinado par redox. A COx de uma substância pode ser utilizada para a determinação da quantidade de elétrons transferíveis nas reações redox e é definida como o número de mols de carga por unidade de massa que um composto é capaz de transferir a um forte oxidante $\left(\mathrm{mol} \mathrm{kg}^{-1}\right)$. Baldotto e colaboradores ${ }^{2}$ estudaram o comportamento redox de amostras de diferentes $\mathrm{AH}$, que após serem tituladas com um oxidante $\left(\mathrm{I}_{2}\right)$ em atmosfera inerte apresentaram valores de ${ }^{\mathrm{F}} \mathrm{E}_{\mathrm{H}}^{\circ}$ semelhantes, entre 0,773 e $0,794 \mathrm{~V}$, a $25^{\circ} \mathrm{C}$. A COx dos $\mathrm{AH}$ variou de 3,88 a 4,39 mol $\mathrm{kg}^{-1}$ a pH 5,0 e de 5,35 até 7,89 mol $\mathrm{kg}^{-1}$ a pH 7,0. Os resultados apresentaram ainda uma correlação positiva e significativa entre a capacidade de oxidação dos ácidos húmicos e as suas concentrações de grupos funcionais fenólicos, quinonas e semiquinonas. Os grupos fenólicos e quinônicos foram indicados como grupos doadores após derivatização dos mesmos e determinação do COx.

A técnica de ultrafiltração proporciona uma avaliação da variabilidade e dinâmica das frações particuladas, causada por processos químicos, físicos e biológicos em ecossistemas aquáticos. $\mathrm{O}$ fracionamento físico ocorre pela diferença de peso molecular em fluxo tangencial por intermédio da ultrafiltração, possibilitando detectar e concentrar compostos orgânicos e inorgânicos em baixas concentrações no meio aquoso. ${ }^{3,4}$ Este método é capaz de processar grandes

*e-mail: marihus@uenf.br volumes de amostras, de forma não destrutiva e livre de reagentes. ${ }^{5}$ Entre as principais frações de material particulado em suspensão encontradas nos sistemas aquáticos, o material ultrafiltrado (MU) é definido operacionalmente com dimensão coloidal entre $1 \mathrm{kD}(\sim$ $0,001 \mu \mathrm{m})$ e $0,1 \mu \mathrm{m}$ e constitui-se, principalmente, de argilominerais e moléculas orgânicas complexas, entre elas ácidos fúlvicos e húmi$\cos .{ }^{3,6}$ As titulações redox das substâncias orgânicas que compõem o MU podem gerar informações úteis para a melhor compreensão da estabilidade dos estoques de $\mathrm{C}$ nos sistemas aquáticos e sua ligação com as trocas entre as biosferas. ${ }^{2,7}$

A estabilidade química do $\mathrm{C}$ junto aos argilominerais ocorre, em geral, com a formação de substâncias húmicas $(\mathrm{SH})$ por meio de um processo genericamente denominado humificação. Este processo pode ser estimado pelo aumento da concentração de radicais livres do tipo semiquinonas (CRLS) nos ácidos húmicos (AH), determinados por meio da ressonância paramagnética eletrônica (RPE). ${ }^{8-11}$ Com o avanço da humificação ocorre a policondensação e a conjugação de estruturas insaturadas nos AH. ${ }^{12}$ Grupamentos fenólicos formados durante o processo de decomposição da matéria orgânica são convertidos em quinonas, via reações de oxidação, as quais são precursoras de radicais livres do tipo semiquinonas nos $\mathrm{AH}$, que se estabilizam com o avanço da humificação. ${ }^{13-15}$ Os radicais livres do tipo semiquinonas dos $\mathrm{AH}$ são grupos doadores de elétrons $\mathrm{s}^{1,16-19} \mathrm{e}$, por meio de titulações redox iodimétricas, podem ser oxidados pelo iodo $\left(\mathrm{I}_{2}\right)$. Usando tais titulações, Baldotto e colaboradores ${ }^{2}$, analogamente à RPE, estimaram o grau de humificação dos AH. Dessa forma, as titulações redox da matéria orgânica (MO) isolada de águas interiores e oceânicas podem fornecer informações qualitativas e quantitativas sobre a MO nesses sistemas naturais e, ainda, sobre a sazonalidade de tais características.

Este estudo teve como objetivo determinar, por meio de titulações redox iodimétricas, a capacidade de substâncias orgânicas isoladas de material ultrafiltrado das águas do Rio Paraíba do Sul transferirem elétrons e relacioná-la com suas características químico-estruturais. 


\section{PARTE EXPERIMENTAL}

\section{Ambiente em estudo}

O estudo envolveu a bacia do Rio Paraíba do Sul, que abrange uma área de aproximadamente $57.000 \mathrm{~km}^{2}$ e uma extensão de 1.145 km, banhando os estados de São Paulo (23,7\%), Minas Gerais $(39,6 \%)$ e Rio de Janeiro $(36,7 \%)$ onde deságua no Oceano Atlântico. ${ }^{6} \mathrm{O}$ rio corre no sentido oeste-leste numa altitude média de $370 \mathrm{~m}$ e seus afluentes originam-se das Serras da Mantiqueira e do Mar. A descarga máxima deste rio atinge, em média, $4.384 \mathrm{~m}^{3} \mathrm{~s}^{-1}$ no período de verão (dezembro a fevereiro) e a descarga mínima se dá no inverno (junho a agosto) com $181 \mathrm{~m}^{3} \mathrm{~s}^{-1}$. $^{6}$

De acordo com Figueiredo e Ovalle, ${ }^{20}$ as classes de vazão no período de estudo, de outubro de 2002 a maio de 2004, apresentaram uma larga faixa de variação, onde períodos de vazão (acompanhando as intensidades de pluviosidade) oscilaram abruptamente (Tabela 1). Situações de baixa e média vazões predominaram no sistema, representando, em média, $42 \%$ do ciclo hidrológico, enquanto foi verificada uma alta vazão em $24 \%$ do período de amostragem.

Tabela 1. Pluviosidade acumulada e classes de vazão nas épocas de amostragem

\begin{tabular}{lcc}
\hline Época & $\begin{array}{c}\text { Pluviosidade Acumu- } \\
\text { lada }(\mathrm{mm})\end{array}$ & Classe de vazão $^{(1)}$ \\
\hline Primavera 2002 & 280 & Média \\
Verão 2002 & 275 & Média \\
Outono 2003 & 230 & Baixa \\
Inverno 2003 & 150 & Baixa \\
Primavera 2003 & 265 & Média \\
Verão 2003 & 390 & Alta \\
Outono 2004 & 180 & Baixa \\
\hline
\end{tabular}

(1) Classes de vazão: Baixa $<600 \mathrm{~m}^{3} \mathrm{~s}^{-1} ; 600 \mathrm{~m}^{3} \mathrm{~s}^{-1}<$ Média $<1.200$ $\mathrm{m}^{3} \mathrm{~s}^{-1} ;$ Alta $>1.200 \mathrm{~m}^{3} \mathrm{~s}^{-1}$.

\section{Amostragem}

As amostras da água superficial do Rio Paraíba do Sul foram coletadas em Campos dos Goytacazes, na latitude $21^{\circ} 45^{\prime} 06^{\prime \prime}$ S e longitude 41'19'32'W, entre outubro de 2002 e maio de 2004, compondo, de acordo com as estações do ano, amostras nas épocas de primavera, verão, outono e inverno.

A coleta da água foi feita sobre uma ponte ( $\mathrm{P}^{\mathrm{te}}$ João Barcelos Martins) em um ponto equiidistante às margens do rio por intermédio de um recipiente de polietileno de aproximadamente $7 \mathrm{~L}$ preso a um cabo, coletando-se os primeiros $50 \mathrm{~cm}$ da coluna d'água. Neste ponto, o rio possui profundidade média de $6 \mathrm{~m}$, seção média de 470 $\mathrm{m}^{2}$ e fluxo instantâneo médio de $715 \mathrm{~m}^{3} \mathrm{~s}^{-1}$, sendo esses valores correspondentes à média de 10 anos de estudo, de 1994 a 2004. ${ }^{6}$ Ainda segundo os autores supracitados, a pluviosidade média durante o período de estudo foi de aproximadamente $80 \mathrm{~mm} \mathrm{mês}^{-1}$.

\section{Ultrafiltração}

Após a coleta, as amostras foram pré-filtradas em cartucho tipo Hollowfiber H5PO1 que excluiu frações com dimensões superiores a $0,1 \mu \mathrm{m}$ e, a seguir, ultrafiltradas através de um cartucho do tipo Spiral S10y1 com retenção de $1 \mathrm{kD}$, ambos da Amicon.

Os sedimentos ultrafiltrados foram solubilizados em uma mistura de $\mathrm{HF}$ e $\mathrm{HCl}$, ambos a 5\% (v/v) por 48 h, visando a remoção de resíduos de minerais de argila e de íons paramagnéticos e, a seguir, transferidos para membranas de diálise de $10 \mathrm{~mL}$ (cut-off $14 \mathrm{kD}$, Thomas Sci.).

As amostras foram submetidas à diálise até a obtenção de condutividade elétrica igual à da $\mathrm{H}_{2} \mathrm{O}$ destilada $\left(1,0 \mu \mathrm{S} \mathrm{cm}^{-1}\right) \mathrm{e}$, a seguir, o material orgânico foi liofilizado e armazenado em dessecador para caracterização (Tabela 2), segundo os procedimentos descritos por Maciel. $^{21}$

\section{Titulações redox}

As titulações redox consistiram da oxidação da MO com I $\mathrm{I}_{2}$ (iodimetria), sob atmosfera inerte. ${ }^{1} \mathrm{O}$ sistema de titulação foi montado utilizando-se um frasco termostatizado e fechado com uma tampa adaptada para conter um eletrodo combinado de $\mathrm{pH}$ ( $\mathrm{pHmetro}$ Quimis Q400A) ou um eletrodo redox combinado (Analyser 6A05-GK AgCl, $\mathrm{Ag} \| \mathrm{Pt}$ ), além de um tubo para entrada de gás argônio (Ar) e uma microbureta de $2 \mathrm{~mL}$ (Gilmont ${ }^{\circledR}$ GS-1200-A, com subdivisões de 2 $\mu \mathrm{L}$ ) (Figura 1). O sistema foi termostatizado através de um banho em recirculação mantido a $25{ }^{\circ} \mathrm{C}$ (Microquímica ${ }^{\circledR}$ Banho MQBCT 99-20).

No preparo do titulado, adicionaram-se ao frasco de titulação $125 \mathrm{~mL}$ de solução tampão fosfato $\left(\mathrm{Na}_{2} \mathrm{HPO}_{4} 0,05 \mathrm{~mol} \mathrm{~L}^{-1} \mathrm{e}\right.$ $\mathrm{NaH}_{2} \mathrm{PO}_{4} \times \mathrm{H}_{2} \mathrm{O} 0,05 \mathrm{~mol} \mathrm{~L}^{-1}$ ) contendo $\mathrm{KCl} 0,05 \mathrm{~mol} \mathrm{~L}^{-1} \mathrm{e}$, em

Tabela 2. Características do material ultrafiltrado (MU) e da sua fração orgânica

\begin{tabular}{|c|c|c|c|c|c|c|c|}
\hline \multirow{2}{*}{ Amostra } & \multicolumn{7}{|c|}{ Características $^{(1)}$} \\
\hline & MU & $\mathrm{N}_{\mathrm{t}}$ & $\mathrm{C}_{\mathrm{t}}$ & $\mathrm{C}: \mathrm{N}$ & ${ }^{15} \mathrm{~N}$ & ${ }^{13} \mathrm{C}$ & $\mathrm{C}_{4}$ \\
\hline Época & $\mathrm{mg} \mathrm{L}^{-1}$ & \multicolumn{2}{|c|}{$-\mathrm{g} \mathrm{kg}^{-1}-$} & & \multicolumn{2}{|c|}{---- \%o ---- } & $\%$ \\
\hline Primavera 2002 & 1,57 & 15,9 & 226,8 & 14,2 & 6,43 & $-22,84$ & 30,25 \\
\hline Verão 2002 & 1,42 & 15,7 & 275,8 & 17,6 & 5,66 & $-22,64$ & 31,25 \\
\hline Outono 2003 & 0,65 & 15,6 & 195,7 & 12,5 & 6,28 & $-22,80$ & 30,49 \\
\hline Inverno 2003 & 0,57 & 15,6 & 175,0 & 11,2 & 6,33 & $-22,20$ & 34,00 \\
\hline Primavera 2003 & 2,09 & 15,8 & 252,4 & 15,9 & 5,73 & $-22,24$ & 34,19 \\
\hline Verão 2003 & 2,24 & 15,7 & 261,1 & 16,7 & 5,63 & $-20,95$ & 42,49 \\
\hline Outono 2004 & 1,40 & 15,6 & 252,8 & 16,2 & 5,36 & $-22,20$ & 34,00 \\
\hline
\end{tabular}

${ }^{(1)}$ Características: $\mathrm{N}_{\mathrm{t}}, \mathrm{C}_{\mathrm{t}}$ e $\mathrm{C}: \mathrm{N}=$ teores totais no $\mathrm{MU}$ de nitrogênio, de carbono e a relação entre tais teores, respectivamente; ${ }^{15} \mathrm{~N},{ }^{13} \mathrm{C}$ e $\mathrm{C}_{4}=$ participação dos isótopos e estimativa de contribuição de plantas com tal tipo de metabolismo na matéria orgânica, respectivamente. 


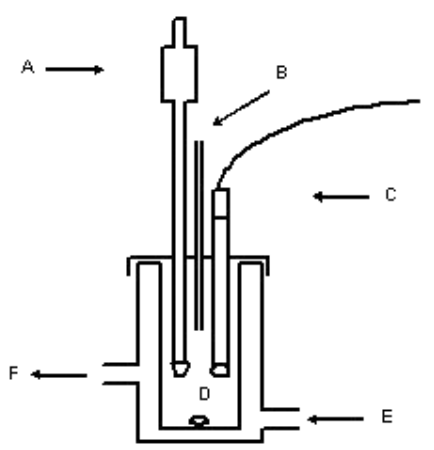

Figura 1. Esquema do equipamento de titulação redox. A: microbureta; B: tubo para entrada de argônio; $C$ : eletrodo de Pt combinado (referência $\mathrm{AgCl}, \mathrm{Ag}$ ); D: agitador magnético; E e F: entrada e saída de água a $25^{\circ} \mathrm{C}$, respectivamente

seguida, ajustou-se o pH com $\mathrm{HCl}$ ou $\mathrm{NaOH}$ para $\mathrm{pH} 5,00$ ou 7,00. A solução foi agitada magneticamente e borbulhada com Ar por 60 min para a depleção de $\mathrm{O}_{2}$. Acrescentou-se $\mathrm{MO}$ à solução para obtenção de uma concentração em suspensão de $50 \mathrm{mg} \mathrm{L}^{-1}$. Novamente, agitou-se e borbulhou-se a suspensão com argônio durante $30 \mathrm{~min}$. Em seguida, ajustou-se o pH da suspensão para 5 ou $7 \mathrm{com} \mathrm{HCl}$ ou $\mathrm{NaOH}$ para o início da titulação.

Para cada titulação, calibrou-se o pHmetro utilizando soluções tampões a pH 4 e 7 . O eletrodo redox foi testado com solução tampão redox férrico-ferrosa, preparada com sulfato ferroso amoniacal 0,100 mol L ${ }^{-1}$, sulfato férrico amoniacal $0,100 \mathrm{~mol} \mathrm{~L}^{-1} \mathrm{e} \mathrm{H}_{2} \mathrm{SO}_{4} 1,00 \mathrm{~mol} \mathrm{~L}^{-1}$. A leitura da f.e. $\mathrm{m}_{\text {cela }}$ em $439 \pm 2 \mathrm{mV}$ indicou o correto funcionamento do eletrodo.22 Padronizou-se a solução de $\mathrm{I}_{2}$, para cada titulação, utilizando tiossulfato de sódio.

Nas titulações, utilizaram-se incrementos de $2 \mu \mathrm{L}$ de solução 0,025 mol kg-1 de $\mathrm{I}_{2}\left(\mathrm{~mol} \mathrm{~kg}^{-1}=\right.$ molinidade $^{1}$, ou seja, o número de mols de $\mathrm{I}_{2}$ por kg de solução) com densidade conhecida e, após cada incremento de titulante, a solução foi agitada e borbulhada com $\mathrm{Ar}$ por mais $30 \mathrm{~s}$ para facilitar a homogeneização do sistema. Em segui-

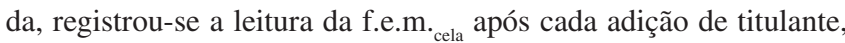
sempre quando a variação no aparelho foi menor ou igual a 2,0 mV $\min ^{-1}$. O ponto final da titulação redox de $\mathrm{AH}$ foi atingido quando

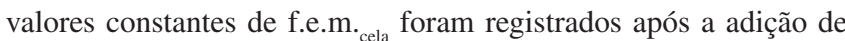
oxidante. Desse ponto em diante, foi iniciada uma nova titulação "de volta" (back titration) com tiossulfato de sódio, cujos procedimentos operacionais foram análogos aos da titulação iodimétrica.

As curvas de titulação redox foram obtidas relacionando-se num gráfico os valores de f.e.m. (eixo das ordenadas) em função da COx da $\mathrm{MO}$ (eixo das abscissas). O valor da $\mathrm{COx}$ foi determinado pelo número de mols de $\mathrm{I}_{2}(\mathrm{aq})$ reduzidos a $\mathrm{I}_{(\mathrm{aq})}^{-}$na reação por unidade de massa de MU ( $\left.\operatorname{mol}_{\mathrm{c}} \mathrm{kg}^{-1}\right)$, usando-se a Equação 1:1

$\mathrm{mol}_{\mathrm{c}} \mathrm{kg}^{-1}=\left(\frac{2 \mathrm{~mol}_{\mathrm{c}}}{\mathrm{mol}_{2}} \times \frac{\text { Densidade da Solução de } \mathrm{I}_{2} \times \text { Molinidade da Solução de } \mathrm{I}_{2}}{\text { Massa de MU }}\right.$

Estimou-se, também, a capacidade de oxidação por massa de carbono nas amostras de MU.

Determinou-se o ponto de inflexão de cada curva de titulação redox, obtendo-se a segunda derivada da função. No ponto de inflexão, a f.e.m. ${ }_{\text {cela }}$ foi estimada por interpolação. ${ }^{1}$ Adicionalmente, realizaramse titulações de amostras em branco, sem adição de MO.

\section{Análise dos dados das titulações redox}

Analisaram-se os dados obtidos com a titulação redox, de acordo com estudo desenvolvido inicialmente por Struyk e Sposito. ${ }^{1}$ Os va- lores de força eletromotriz (f.e.m. ${ }_{\text {.ela }}$ ), medidos durante as titulações redox, foram relacionados com o potencial formal do eletrodo $\left(\mathrm{F}_{\mathrm{H}}\right)$ de acordo com a Equação 2:

f.e.m. cela $={ }^{\mathrm{F}} \mathrm{E}_{\mathrm{H}}\left(\mathrm{MO}_{\mathrm{Ox}}, \mathrm{MO}_{\mathrm{Red}}\right)-\mathrm{E}_{\mathrm{H}}^{\mathrm{o}}(\mathrm{AgCl}, \mathrm{Ag})$

onde $\mathrm{E}_{\mathrm{H}}{ }^{\circ}(\mathrm{AgCl}, \mathrm{Ag})=222 \mathrm{mV}$ é o potencial padrão do eletrodo de referência $\left(\mathrm{AgCl}, \mathrm{Ag}\right.$ a $25^{\circ} \mathrm{C}$ ) relativo ao eletrodo padrão de hidrogênio $e^{\mathrm{F}} \mathrm{E}_{\mathrm{H}}\left(\mathrm{MO}_{\mathrm{ox}}, \mathrm{MO}_{\text {red }}\right)$ é o potencial formal de eletrodo da meia-reação $\mathrm{MO}_{\mathrm{O}}, \mathrm{MO}_{\mathrm{Red}}$, que correspondem, respectivamente, às formas oxidada e reduzida da $\mathrm{MO}$, de acordo com a reação geral de redução:

$\mathrm{MO}_{\mathrm{Ox}}+\mathrm{ne}^{-}+\mathrm{qH}^{+}=\mathrm{MO}_{\text {Red }}$

A Equação 3 indica que a reação de redução da MO consome, concomitantemente, $n$ elétrons e q prótons. Assim, a relação q/n que indica o número de moles de prótons (q) por moles de elétrons (n) transferidos durante a oxidação de $1 \mathrm{~mol}$ de sítios redox da MO, estimada pela redução de $1 \mathrm{~mol} \mathrm{de} \mathrm{I}_{2}$, foi calculada utilizando-se os valores da f.e.m. cela $_{\text {, }}$ obtidos para dois valores de $\mathrm{pH}(5,0$ e 7,0$)$, por meio da Equação 4:

$-\frac{\mathrm{q}}{\mathrm{n}}=\frac{\frac{\Delta \text { f.e.m. }}{0,05916}}{\Delta \mathrm{pH}}$

onde $\Delta$ f.e.m. é a variação da f.e.m. cela $_{\text {p }}$ por unidade log de variação do $\mathrm{pH}(\Delta \mathrm{pH})$ no ponto de inflexão da titulação.

No ponto de inflexão, de acordo com a Equação de Nernst, ${ }^{1,23}$ calculou-se o ${ }^{\mathrm{F}} \mathrm{E}_{\mathrm{H}}^{\mathrm{o}}$ para a meia reação redox $\left(\mathrm{MO}_{\mathrm{Ox}}, \mathrm{MO}_{\mathrm{Red}}\right)$, usandose a Equação 5:

f.e.m. cela $={ }^{\mathrm{F}} \mathrm{E}_{\mathrm{H}}^{\mathrm{o}}\left(\mathrm{MO}_{\mathrm{Ox}}, \mathrm{MO}_{\text {Red }}\right)-(\mathrm{q} / \mathrm{n}) 0,05916 \mathrm{pH}-\mathrm{E}_{\mathrm{H}}^{\mathrm{o}}(\mathrm{AgCl}, \mathrm{Ag})$

na qual, ${ }^{\mathrm{F}} \mathrm{E}_{\mathrm{H}}^{\mathrm{o}}\left(\mathrm{MO}_{\mathrm{Ox}}, \mathrm{MO}{ }_{\mathrm{Red}}\right)$ indica o potencial formal padrão do eletrodo.

$\mathrm{O}^{\mathrm{F}} \mathrm{E}_{\mathrm{H}}{ }^{\circ}$ de cada amostra de $\mathrm{MO}$ foi convertido na constante de equilíbrio condicional usando-se a seguinte relação: ${ }^{23}$

$\log \mathrm{K}_{\mathrm{c}}=\frac{{ }^{\mathrm{F}} \mathrm{E}_{\mathrm{H}}^{\mathrm{o}}}{0,05916}$

na qual $\mathrm{K}_{c}$ é a constante de equilíbrio condicional, a $298 \mathrm{~K}$, correspondendo à reação de redução genérica para um elétron transferido: ${ }^{1}$

$\mathrm{m} \mathrm{Ox}+(\mathrm{q} / \mathrm{n}) \mathrm{H}^{+}+\mathrm{e}^{-}=\mathrm{p}$ Red

$\mathrm{O}$ pE para o par redox $\mathrm{MO}_{\mathrm{Ox}}, \mathrm{MO}_{\text {Red }}$ foi obtido conforme a Equação 8:23

$\mathrm{pE}=\log \mathrm{Kc}-(\mathrm{q} / \mathrm{n}) \mathrm{pH}$

A variação de energia livre padrão de Gibbs $\left(\Delta \mathrm{G}^{\circ}\right)$ para a semireação de redução da MO estudada foi estimada pelas equações obtidas de acordo com a primeira e a segunda leis da termodinâmica:

$\Delta \mathrm{G}^{\mathrm{o}}=\mathrm{nF}^{\mathrm{F}} \mathrm{E}_{\mathrm{H}}^{\mathrm{o}}$

na qual, $\mathrm{F}=$ constante de Faraday, igual a 23,061 kcal V $\mathrm{mol}^{-1}$.

\section{Análises estatísticas}

Estimou-se o desvio-padrão da média para cada variável experimental e realizou-se o teste de agrupamento pela variância 
mínima e distância euclidiana, usando-se a análise multivariada dos dados. ${ }^{24}$

\section{RESULTADOS E DISCUSSÃO}

\section{Potencial do eletrodo}

A Figura 2 mostra um exemplo de uma curva de titulação redox iodimétrica. Tais curvas de titulação potenciométrica dos AH foram semelhantes às obtidas por Struyk e Sposito, ${ }^{1}$ usando três amostraspadrão de AH da IHSS (International Humic Substance Society), e às apresentadas por Baldotto e colaboradores ${ }^{2}$, usando amostras de

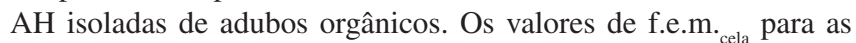
titulações redox realizadas a pH 5 e 7 e os resultados dos cálculos de ${ }^{\mathrm{F}} \mathrm{E}_{\mathrm{H}}^{\mathrm{o}}, \log \mathrm{K}_{\mathrm{c}}, \mathrm{pe}_{\mathrm{c}}$ encontram-se na Tabela 3.

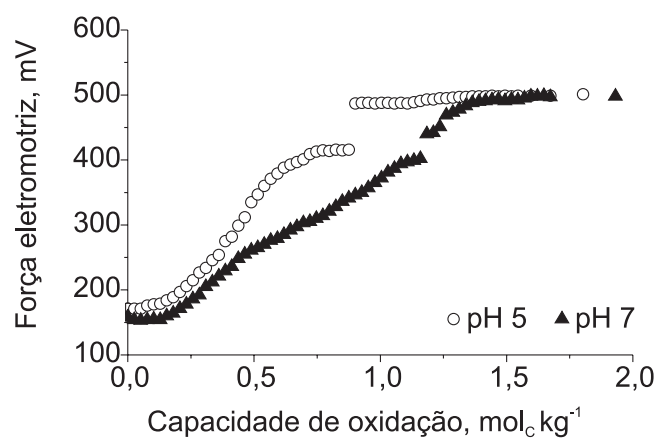

Figura 2. Titulações redox da matéria orgânica isolada do material ultrafiltrado do Rio Paraíba do Sul. Amostras coletadas no inverno de 2003 e solubilizadas em solução tampão de fosfatos $\left(\mathrm{NaH}_{2} \mathrm{PO}_{4}\right.$ e $\mathrm{Na}_{2} \mathrm{HPO}_{4} \mathrm{O}, 05 \mathrm{~mol}$ $L^{-1}$, contendo $\mathrm{KCl}$ 0,05 mol L-1, a pH 5,00 e a pH 7,00)

No presente estudo, os valores de ${ }^{\mathrm{F}} \mathrm{E}_{\mathrm{H}}{ }^{\circ}$ para a semi-reação de redução dos $\mathrm{AH}$ pouco variaram, apresentando valores entre 0,754 e 0,786 V. Struyk e Sposito, ${ }^{1}$ usando três amostras de AH obtidas de solo e de sedimentos, encontraram valores de ${ }^{\mathrm{F}} \mathrm{E}_{\mathrm{H}}{ }^{\mathrm{o}}$ próximos a 0,780 $\mathrm{V}$ e Baldotto e colaboradores ${ }^{2}$, estudando alguns adubos orgânicos, obtiveram valores em torno de $0,782 \mathrm{~V}$.

Em ambos os trabalhos supracitados foram usadas soluções aquosas de $\mathrm{I}_{2}$ como oxidante. Tais $\mathrm{AH}$ estudados apresentaram ${ }^{\mathrm{F}} \mathrm{E}_{\mathrm{H}}{ }^{\circ}$ e $\log \mathrm{K}_{\mathrm{c}}$ análogos aos de compostos quinonas. ${ }^{16}$ No entanto, essas substâncias se diferenciaram pela mais ampla relação $q / n$ verificada nos compostos quinonas $(1,00)$ em relação aos $\mathrm{AH}(0,33)$, valores também verificados para a MO deste estudo.
Uma comparação entre os valores das propriedades redox de alguns pares comuns nos sistemas naturais realizada por Struyk e Sposito $^{1}$ e por Baldotto e colaboradores, ${ }^{2}$ permitiu alocar o par redox $\mathrm{MO}_{\mathrm{Ox}}, \mathrm{MO}_{\mathrm{Red}}$ na "seqüência redox" (Tabela 4) indicada por Stumm e Morgan. ${ }^{23}$ Os $\mathrm{AH}$ podem reduzir $\mathrm{Hg}(\mathrm{II})$ e $\mathrm{Mn}(\mathrm{IV})$, conforme observaram Alberts e colaboradores ${ }^{25}$ e Sunda e Kieber, ${ }^{26}$ respectivamente, incubando tais íons metálicos com bactérias redutoras e AH. No presente estudo, tal comparação também foi realizada para a MO isolada do material ultrafiltrado do Rio Paraíba do Sul e, dada a semelhança entre os valores das suas propriedades redox com as dos estudos de Struyk e Sposito ${ }^{1}$ e Baldotto e colaboradores, ${ }^{2}$ podem ser obtidas as mesmas inferências quanto à sua reatividade nos sistemas naturais. Tal fato torna-se fundamental na discussão de resultados como os apresentados por Almeida e colaboradores, ${ }^{6}$ que encontraram uma variação entre 93 e $290 \mathrm{ng} \mathrm{g}^{-1}$ nas concentrações de $\mathrm{Hg}$ do Rio Paraíba do Sul, cujos picos de maior e de menor concentração de tal elemento foram determinados em novembro de 1999 e em agosto de 2000, respectivamente.

\section{Capacidade redox}

Os valores da COx (Tabela 5), em termos de massa de MU, variaram de 0,11 a $0,19 \mathrm{~mol}_{\mathrm{c}} \mathrm{kg}^{-1}$ a pH 5 e de 0,17 a $0,32 \mathrm{~mol} \mathrm{~kg}^{-1}$ a $\mathrm{pH} 7,0$. Corrigindo tais valores da COx para a massa de carbono do MU (em média de 234,2 $\mathrm{g} \mathrm{kg}^{-1}$ de C), observou-se que as suas magnitudes oscilaram de 0,48 a $0,85 \mathrm{~mol} \mathrm{~kg}^{-1}$ a pH 5 e de 0,76 a $1,19 \mathrm{~mol} \mathrm{~kg}^{-1}$ a pH 7,0.

Valores de COx e variações com o aumento do $\mathrm{pH}$ semelhantes têm sido obtidos por outros autores. Struyk e Sposito ${ }^{1}$ determinaram valores de COx entre 1,09 e 6,50 mol $\mathrm{kg}^{-1}$ a pH 5 e variando de 3,30 até 11,50 mol $\mathrm{kg}^{-1}$ a pH 7 para AH isolados de amostras-padrão da IHSS. Baldotto e colaboradores, ${ }^{2}$ para AH obtidos de adubos orgânicos, verificaram COx de 3,88 a 4,39 mol $\mathrm{kg}^{-1}$ a pH 5 e de 5,35 a 7,89 mol $_{\mathrm{c}} \mathrm{kg}^{-1}$ a pH 7. Matthiessen, ${ }^{17} \mathrm{em}$ amostras de $\mathrm{AH}$ sintético, encontrou valores médios de 7,5 mol $\mathrm{kg}^{-1}$ a pH 5,0 e de 10,5 mol $\mathrm{kg}^{-1}$ a pH 7,0. Tais variações da COx com o pH são típicas dessas reações de redução que consomem prótons e podem ser entendidas conforme a Equação 7.

Nota-se que a COx do material ultrafiltrado do Rio Paraíba do Sul foi bem inferior àquelas obtidas para $\mathrm{AH}$ pelos autores supracitados, em ambos os valores de $\mathrm{pH}$. Como o material ultrafiltrado, neste estudo, foi obtido a partir de sedimentos em suspensão, admite-se que possua maior contribuição de frações solúveis da matéria orgânica e, consequientemente, menor COx. Tal propriedade foi associada ao aumento da estabilidade de substâncias humificadas da matéria orgânica. ${ }^{2}$ Dessa forma, nas estações do ano onde houve maior COx

Tabela 3. Propriedades redox ${ }^{(1)}$ da matéria orgânica isolada do material ultrafiltrado (MU)

\begin{tabular}{|c|c|c|c|c|c|c|c|c|}
\hline \multirow{2}{*}{ Amostra } & \multicolumn{2}{|c|}{ f.e.m $m_{\text {cela }}$} & \multirow{2}{*}{$\mathrm{q} / \mathrm{n}$} & \multirow{2}{*}{${ }^{\mathrm{F}} \mathrm{E}_{\mathrm{H}}^{\circ}$} & \multirow{2}{*}{$\log \mathrm{K}_{\mathrm{c}}$} & \multicolumn{2}{|c|}{$\mathrm{pE}_{\mathrm{c}}$} & \multirow{2}{*}{$\Delta \mathrm{G}^{\circ}$} \\
\hline & $\mathrm{pH} 5$ & $\mathrm{pH} 7$ & & & & $\mathrm{pH} 5$ & $\mathrm{pH} 7$ & \\
\hline Estação & \multicolumn{2}{|c|}{$\mathrm{mV}$} & & V & & & & $\mathrm{kcal} \mathrm{mol}^{-1}$ \\
\hline Primavera 2002 & $452 \pm 3$ & $418 \pm 1$ & 0,29 & 0,759 & 12,8 & 11,4 & 10,8 & $-17,5$ \\
\hline Verão 2002 & $467 \pm 1$ & $429 \pm 1$ & 0,33 & 0,771 & 13,3 & 11,7 & 11,0 & $-18,1$ \\
\hline Outono 2003 & $452 \pm 1$ & $420 \pm 1$ & 0,27 & 0,786 & 12,7 & 11,4 & 10,9 & $-17,4$ \\
\hline Inverno 2003 & $457 \pm 1$ & $420 \pm 1$ & 0,31 & 0,776 & 13,1 & 11,5 & 10,8 & $-17,8$ \\
\hline Primavera 2003 & $455 \pm 1$ & $425 \pm 3$ & 0,25 & 0,754 & 12,7 & 11,4 & 10,9 & $-17,3$ \\
\hline Verão 2003 & $457 \pm 1$ & $420 \pm 3$ & 0,31 & 0,764 & 13,0 & 11,5 & 10,9 & $-17,7$ \\
\hline Outono 2004 & $458 \pm 3$ & $418 \pm 2$ & 0,34 & 0,772 & 13,2 & 11,5 & 10,8 & $-18,0$ \\
\hline
\end{tabular}

${ }^{(1)}$ Propriedades redox: definidas na seção Parte Experimental. As médias experimentais estão acompanhadas pelo seu erro-padrão. 
Tabela 4. Propriedades redox de alguns pares comuns em sistemas naturais

\begin{tabular}{|c|c|c|c|c|}
\hline Pares redox & $\begin{array}{c}{ }^{\mathrm{F}} \mathrm{E}_{\mathrm{H}}{ }^{\circ} \\
\mathrm{V}\end{array}$ & $\log K_{c}$ & $\mathrm{pE}_{\mathrm{c}}^{(1)}(\mathrm{pH} 5)$ & $\mathrm{pE}_{\mathrm{c}}(\mathrm{pH} 7)$ \\
\hline $1 / 2 \mathrm{MnO}_{2}(\mathrm{~s})+2 \mathrm{H}^{+}+\mathrm{e}^{-}=1 / 2 \mathrm{Mn}^{2+}(\mathrm{aq})+\mathrm{H}_{2} \mathrm{O}$ & 1,23 & 20,8 & 10,8 & 6,8 \\
\hline $\mathrm{Fe}(\mathrm{OH})^{2+}(\mathrm{aq})+\mathrm{e}^{-}+\mathrm{H}^{+}=\mathrm{Fe}^{2+}(\mathrm{aq})+\mathrm{H}_{2} \mathrm{O}$ & 0,90 & 15,2 & 10,2 & 8,2 \\
\hline $1 / 2 \mathrm{Hg}^{2+}(\mathrm{aq})+\mathrm{e}^{-}=1 / 2 \mathrm{Hg}^{\mathrm{o}}(\mathrm{g})$ & 0,85 & 14,4 & 14,4 & 14,4 \\
\hline $\mathrm{MO}_{\mathrm{ox}}(\mathrm{aq})+1 / 3 \mathrm{H}^{+}+\mathrm{e}^{-}=\mathrm{MO}_{\mathrm{red}}(\mathrm{aq})$ & 0,77 & 13,0 & 11,5 & 10,9 \\
\hline $\mathrm{FeOOH}(\mathrm{s})+\mathrm{e}^{-}+3 \mathrm{H}^{+}=\mathrm{Fe}^{2+}(\mathrm{aq})+2 \mathrm{H}_{2} \mathrm{O}$ & 0,77 & 13,0 & 2,0 & $-4,0$ \\
\hline $\mathrm{Fe}^{3+}(\mathrm{aq})+\mathrm{e}^{-}=\mathrm{Fe}^{2+}(\mathrm{aq})$ & 0,77 & 13,0 & 13,0 & 13,0 \\
\hline
\end{tabular}

(1) Os valores de $\mathrm{pE}$ foram calculados usando-se os valores de $\log \mathrm{K}_{\mathrm{c}}$, concentração de redutores e oxidantes $=10^{-4} \mathrm{~mol} \mathrm{~L}^{-1}$ de espécies solúveis, atividade das fases sólidas iguais a 1 e $\mathrm{P}_{\mathrm{O} 2}=0,21 \mathrm{~atm}$. Os valores em negrito são as médias dos dados obtidos com a matéria orgânica (MO) isolada do material ultrafiltrado do Rio Paraíba do Sul.

Tabela 5. Capacidades de oxidação (COx) e de redução (CRed) da matéria orgânica corrigidas para as massas de MU e de carbono no sedimento

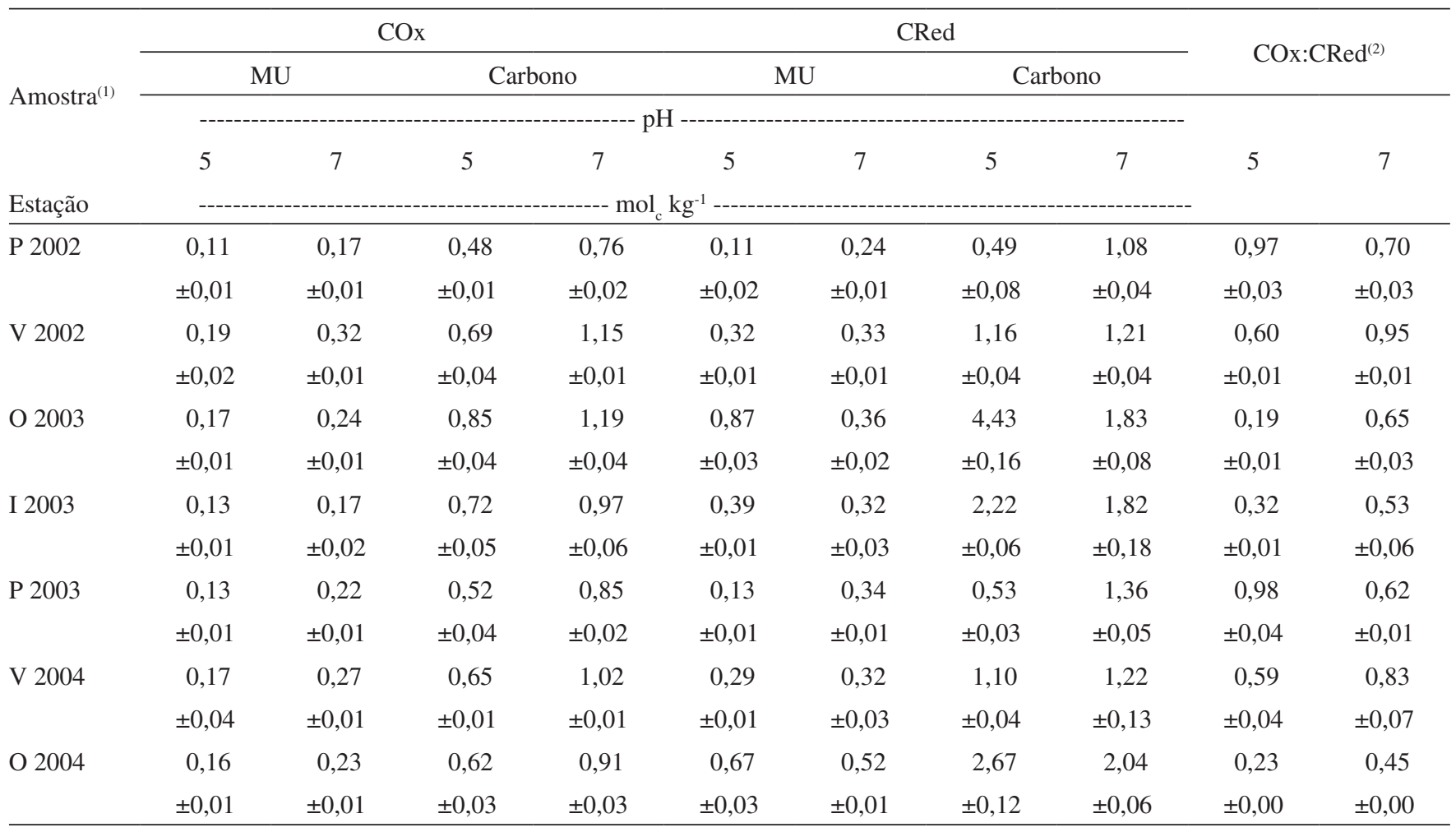

(1) Amostra: P, V, O e I = primavera, verão, outono e inverno; ${ }^{(2)}$ relação por massa de carbono no material ultrafiltrado; As médias experimentais estão acompanhadas pelo seu desvio-padrão.

da matéria orgânica do material ultrafiltrado, poderia se esperar maior contribuição das formas mais estáveis de carbono. A COx da matéria orgânica permitiu separar tais situações, assumindo que quanto maior seu valor, mais elevada é a concentração de grupos aromáticos do tipo quinonas, já associados à maior estabilidade da matéria orgânica. ${ }^{2,8,9,13}$

A COx do material ultrafiltrado do Rio Paraíba do Sul variou com a pluviosidade e a vazão. Pode-se inferir, ainda, que processos de transferência de carbono advindos da bacia do Rio Paraíba do Sul contribuam com a incorporação de formas de carbono em seus sedimentos de maneira distinta conforme a estação do ano. Dentre tais formas, incluem-se as substâncias húmicas, principalmente ácidos fúlvicos.

Para se testar a reversibilidade da reação redox envolvendo a MO do sedimento ultrafiltrado do Rio Paraíba do Sul, após a titulação com $\mathrm{I}_{2}$ obteve-se back titration usando-se o $\mathrm{NaS}_{2} \mathrm{O}_{7}$ (Tabela 5). Neste estudo, tal capacidade de reversibilidade da $\mathrm{MO}$ ao estado reduzido foi denominada de capacidade de redução (CRed) e seus valores foram estimados (Tabela 5) visando uma compreensão desses resultados. Os valores da CRed, em termos de massa de MU, variaram de 0,11 a $0,87 \mathrm{~mol}_{\mathrm{c}} \mathrm{kg}^{-1}$ a pH 5 e de 0,24 a $0,52 \mathrm{~mol}_{\mathrm{c}} \mathrm{kg}^{-1}$ a $\mathrm{pH}$ 7,0. Corrigindo tais valores da COx CRed para a massa de carbono do MU (em média de 234,2 $\mathrm{g} \mathrm{kg}^{-1}$ de C), observou-se que as suas magnitudes oscilaram de 0,49 a $4,43 \mathrm{~mol}_{\mathrm{c}} \mathrm{kg}^{-1}$ a pH 5 e de 1,08 a 2,04 mol $\mathrm{kg}^{-1}$ a pH 7,0. Para todas as amostras, a CRed foi superior à $\mathrm{COx}$, indicando que a MO das amostras do MU do Rio Paraíba do Sul já estavam parcialmente oxidadas no momento da titulação iodimétrica.

\section{Análise multivariada dos dados}

O dendograma, obtido por meio da análise multivariada, ${ }^{24}$ indicou que o conjunto de dados pode ser dividido em dois grupos mais distantes, representados pelas estações inverno e verão, que são também 
aquelas que apresentam as maiores discrepâncias de pluviosidade e vazão (Figura 3).

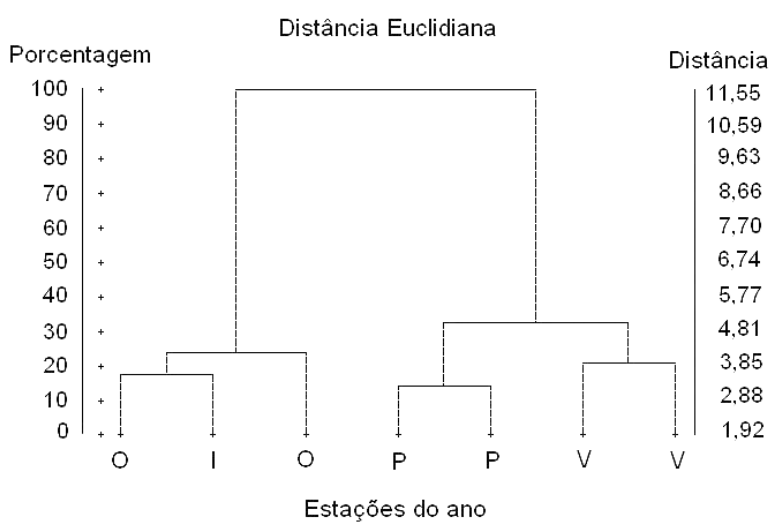

Figura 3. Agrupamento pela variância mínima e distância euclidiana usandose a análise multivariada dos dados. P, V, O e I: estações do ano primavera, verão, outono e inverno, respectivamente. Foram usadas 20 variáveis obtidas para o conjunto de amostras estudadas

Na época de alta vazão (ocorrências de chuva de verão subtropical), que compreende geralmente os meses de dezembro a março, verificou-se a maior concentração de material orgânico dissolvido e de material particulado em suspensão. Estes resultados são atribuídos ao escoamento superficial, à interação de áreas alagáveis com o rio, à erosão de solos (principalmente utilizado para prática agrícola) e remobilização do sedimento de fundo, promovendo um maior transporte de material no canal fluvial do Rio Paraíba do Sul. Tal concentração de materiais em suspensão, de forma geral, foi acompanhada por uma maior atividade redox da MO. Como já foi discutido, o aumento nessa atividade está relacionado com uma maior estabilidade das formas de carbono. Assim, é possível inferir que o transporte de material terrestre durante o verão concentra, além da MO solúvel (por exemplo, ácidos fúlvicos), materiais estabilizados na fração argila dos solos, ou seja, aqueles com maior condensação ou grau de humificação. De maneira geral observa-se que a vazão apresentou variação sazonal e interanual e, concomitantemente, foi modificada a atividade química da MO nesses sistemas.

\section{CONCLUSÕES}

Neste trabalho concluiu-se que a COx da MO obtida do MU do Rio Paraíba do Sul variou com o pH do sistema e com a estação do ano. A COx verificada para tais materiais, por massa de carbono no material ultrafiltrado, foi de 0,48 a $0,85 \mathrm{~mol}_{\mathrm{c}} \mathrm{kg}^{-1}$ a pH 5,0 e de 0,76 a $1,19 \mathrm{~mol}_{\mathrm{c}} \mathrm{kg}^{-1}$ a pH 7,0. Os valores do ${ }^{\mathrm{F}} \mathrm{E}_{\mathrm{H}}{ }^{\circ}$ para a semi-reação de redução do par redox $\mathrm{MO}_{\mathrm{Ox}}, \mathrm{MO}_{\mathrm{Red}}$ foram semelhantes entre as estações do ano em que foram coletadas as amostras de material ultrafiltrado do Rio Paraíba do Sul, sendo em média de 0,768 V. A MO isolada do material ultrafiltrado do Rio Paraíba do Sul apresentou modificações em sua atividade redox de acordo com a época de amostragem, devido principalmente à presença de materiais em suspensão que são transportados durante as estações chuvosas na coluna d'água.

\section{AGRADECIMENTOS}

O presente trabalho contou com auxílios dos projetos: (CNPq/ CTHidro Proc. 556160/2006-1/151856/2007-9), Instituto do Milênio Estuários - (CNPq Proc. 420050/2005-1), da Cooperação BrasilAlemanha POLCAMAR: (CNPq/BMBF Proc. 590002/2005-8) e (FAPERJ Proc. E-26/153.086/2006 e E-26/110.633/2007).

\section{REFERENCIAS}

1. Struyk, Z.; Sposito, G.; Geoderma 2001, 102, 329.

2. Baldotto, M. A.; Canellas, L. P.; Canela, M. C.; Simões, M. L.; MartinNeto, L.; Fontes, M. P. F.; Velloso, A. C. X.; R. Bras. Ci. Solo 2007, 3, 465.

3. Hedges, J. I.; Cowie, G. L.; Richey, J. E.; Quay, P. D.; Benner, R.; Strom, M.; Forsberg, B. R.; Limnol. Oceanogr. 1994, 39, 743.

4. Ludwig, U.; Grischek, T.; Neitzel, P.; Nestler, W.; J. Chromatogr. 1997, 763,315 .

5. Young, C.; Water Res. 1999, 33, 3056.

6. Almeida, M. G.; Rezende, C. E.; Souza, C. M. M.; Geochim. Brasil. 2007, 21, 111.

7. Machado, P. L. O. de A.; Quim. Nova 2005, 28, 329.

8. Martin-Neto, 1.; Andriulo, A. E.; Traghetta, D.; Soil Sci. 1994, 157, 365.

9. Martin-Neto, L.; Vieira, E. M.; Sposito, G.; Environ. Sci. Technol. 1994, $28,1867$.

10. Saab, S. C.; Martin-Neto, L.; Quim. Nova 2003, 26, 497.

11. Pérez, M. G.; Martin-Neto, L.; Saab, S. C.; Novotny, E. H.; Milori, D. M. B. P.; Bagnato, V. S.; Colnago, L. A.; Melo, W. J.; Knicker, H.; Geoderma 2004, 118, 181.

12. Piccolo, A.; Soil Sci. 2001, 166, 810.

13. Milori, D. M. B. P.; Martin-Neto, L.; Bayer, C.; Mielniczuck, J.; Bagnato, V. S.; Soil Sci. 2002, 167, 739.

14. Budziak, C. R.; Maia, C. M. B. F.; Mangrich, A. S.; Quim. Nova 2004, $27,399$.

15. Rivero, C.; Chirenge, T.; Ma, L. Q.; Martinez, G.; Geoderma 2004, 123, 355.

16. Helburn, R. S.; Maccarthy, P.; Anal. Chem. Acta 1994, 295, 263.

17. Matthiessen, A.; Vom Wasser 1995, 84, 229.

18. Lovley, D. R.; Coates, J. D.; Blunt-Harris, E. L.; Phillips, E. J. P.; Woodward, J. C.; Nature 1996, 382; 445.

19. Scott, D.T.; Mcknight, D.M.; Harris, E.; Kolesar, S.; Lovley, D.; Environ. Sci. Technol. 1998, 32, 2984.

20. Figueiredo, R. O.; Ovalle, A. R. C.; Water, Air and Soil Pollut. 1998, 105, 399 .

21. Maciel, M. S.; Monografia em Ciências Biológicas, Universidade Estadual do Norte Fluminense Darcy Ribeiro, Brasil, 2005.

22. Light, T. S.; Anal. Chem. 1972, 44, 1038.

23. Stumm, W.; Morgan, J. J.; Aquatic Chemistry: Chemical Equilibria and Rates in Natural Waters, $3^{\text {rd }}$ ed., Wiley-Interscience: New York, 1996.

24. Cruz, C. D.; Análise multivariada e simulação, Editora UFV: Viçosa, 2006.

25. Alberts, J. J.; Schindler, J. E.; Miller, R. W.; Science 1974, 184, 895.

26. Sunda, W. G.; Kieber, D. J.; Nature 1994, 367, 62. 